\title{
Improved YARN resource scheduling algorithm based on network load sensing
}

\author{
Xuyang Ding ${ }^{1}$, Xiaohui Liu ${ }^{2, a}$, Rui Zhang ${ }^{3}$, Shan Tang ${ }^{3}$, Linqin Sun ${ }^{3}$ and Ying Xie ${ }^{4}$ \\ ${ }^{1}$ Powerchina Chengdu Engineering Corporation Limited, Chengdu 610072, China \\ ${ }^{2}$ National Computer Network Emergency Response Coordination Center, 100029Beijing, China \\ ${ }^{3}$ University of Electronic Science and Technology of China, Chengdu 611731, China \\ ${ }^{4}$ Southwest University For Nationalities, Chengdu 610041, China
}

\begin{abstract}
Confronted with multitask scheduling, YARN may conduct a precise calculation and allocation towards every single task's computing and memory resources,however it failed to consider the impact on task fulfillment and cluster resources utilization. In this paper, we propose a new algorithm to allocate the data interconnected tasks to the same node or to another closely-located low network load node. This new algorithm firstly builds a task flow graph for the task requests of an application, and then assigns tasks more properly using the greedy strategy, based on the state of network resource. In this way, it significantly improves the management performance of YARN in the case of network congestion or instability. Besides, it consumesless execution time, makes higher utilization of CPU and requires lower network load.
\end{abstract}

Keywords: multitask scheduling; YARN; task flow graph; greedy strategy; network resource.

\section{Introduction}

Distributed computing cluster integrates the CPU, memory and disk space resources, and deals with massive data using distributed applications to mobilize the resources on the cluster. In order to free programmers from the complex distributed task scheduling and synchronization, a series of computational frameworks for different scenarios are introduced, such as Spark for Memory oriented iterative computing, Storm for flow calculation, machine learning framework Mahout and open source software framework Map Reduce[1,2].

Map Reduce can be used to deal with large-scale data through the realization of Map Reduce programming paradigm, which can split applications into a number of small tasks to run on any node in the cluster. Map Reduce cluster resource management, mainly includes CPU resources and memory resources, providing the operating system level support for a variety of computing frameworks through the resource management system YARN.

YARN resource manager adopts the structure of master and slave, which mainly includes Resource Manager (RM), Node Manage (NM) and Application Master (AM). Although YARN can provide reliable and efficient resource management support for distributed computing framework and distributed applications on the operating system level, it only supports CPU and memory in the aspect of resources scheduling and isolation. In the process of task execution, it can't be ignored that the

\footnotetext{
${ }^{a}$ Corresponding author : lxh@cert.org.cn
} 
network IO resources exert a great impact on the efficiency of the task execution due to the communication requirements among tasks. For example, in the Map Reduce computing framework, since Reduce tasks need to receive calculated results from map tasks for further computation, if the network transmission quality between the map and reduce tasks cannot be guaranteed, the task completion time will increase greatly. For another example, YARN assigns task to a node as close to last task node as possible in the case allowed by computational and memory resources. In this mode, if the first task is assigned to network congestion regions in the cluster, the following tasks can be possibly assigned in the same regions which further increase congestion.

This paper proposes an improved YARN resource management mechanism supporting network IO resource scheduling. It firstly builds a task flow graph for the task requests of an application, and then can be able to assign tasks more properly using the greedy strategy, according to all requests combined with the state of CPU, memory and network resource. In this way, it significantly improves the management performance of YARN in the case of network congestion or instability.

\section{Related work}

The main function of YARN is resource management. It supports FIFO, Scheduler Fair and Scheduler Capacity strategy and currently there are many researches focusing on optimizing the YARN scheduling strategy. A dynamical resource scheduling strategy based on adaptability and urgency was proposed by Yi Yao[3], which improved the resource utilization and reduced task completion time compared with FIFO, Fair Scheduler and other built-in algorithms. Kapil presented a method to allocate CPU and memory resources based on the current cluster load and to-be-assigned task workload[4], and had great improvements in performance compared to the FIFO strategy. Some others[5] proposed resource dynamic reservation scheduling strategy to avoid YARN resource deadlock problem, which collected each active node resources occupancy (mainly computing resources and storage resources) and maintained a queue of tasks waiting for resource allocation. The above method mainly made some improvements to the FIFO or Fair Scheduler strategy, but were still limited by computing and memory resources scheduling, and did not take network load IO into account as an important resource.

With the demand of network IO resource allocation in distributed system becoming higher and higher [6], network IO resource scheduling and isolation works based on YARN is under way in the open source community[7]. Yazdanov indicated that development of network isolation was far inferior to CPU isolation and memory isolation in the cloud platform [8], and proposed resource scheduling algorithm according to the available bandwidth of the network. The algorithm detected network resource occupation and controlled the number of tasks in parallel to ensure completion time under different network bandwidth. Bhuvan focused on the CPU and network bandwidth modeling [9], and put forward an efficient resource control mechanism through the CPU and network available bandwidth prefetching and dynamic allocation combined with network isolation based on historical records. Other researchers focused on the global study of YARN resource scheduling, in which computation resource, memory resource and network IO resource were allocated simultaneously. Chen proposed the data localization optimization algorithm [10], which calculated the data location in the process of resource scheduling through the establishment of data interference weight mathematical model. In the algorithm, data location optimization was conducive to reducing the data transmission delay. Qing proposed a method to calculate the number of sub tasks and assignment priority index for allocating resources [11], which could balance resources and tasks. Grandl introduced the heuristic method and proposed the multi-type resources scheduling algorithm [12]. The algorithm not only took into account of the computing resources and memory resources, storage resources and network resources, at the same time, gave assignments based on multi-type resources demand to prevent resource fragmentation and excessive allocation problem. In this paper, we mainly consider the high network load into the resource allocation strategy, and strive to maximize the allocation of resources and shorten the task time in the context of high network load. 


\section{Improved YARN resource management mechanism supporting network 10 resource scheduling}

When the resource scheduler YARN schedules multiple tasks, it makes a precise calculation and allocation of computing resources and memory resources for each task. Although various types of tasks are divided to accomplish different tasks, there are usually some data exchanges between different types of tasks. For example, in the Map Reduce framework, Map task and Reduce task are different tasks, but the input data of the Reduce task is the output data of the Map task. In spite of the fact that YARN considers the problem and assign data interaction task to the same node or a similar node as close as possible, it still can't solve the problem fundamentally. Firstly, YARN can not accurately determine the correlation of data interaction between tasks by task priority or by other heuristic algorithms. Secondly, this algorithm does not consider the scenario of network congestion. If the first task is assigned to network congestion regions in the cluster, the following tasks can be either allocated in the same region to aggravate the network congestion, or allocated dispersedly which is not conducive to the rapid completion of the application task.

To solve the above two problems, this paper proposes a resource scheduling algorithm supporting network IO resource allocation, which mainly includes two aspects below:

(1) Task set partitioning: modeling the user's task request, and dividing the tasks into different task subsets according to their communication dependencies.

(2) Resource scheduling: based on the task partition results, calculating the priority queue and scheduling for all tasks combined with the network IO resources in accordance with the greedy strategy.

\subsection{System model}

Definition 1: Cluster environment is defined as a quintet $\mathbf{E}$. E=(Node, $\mathbf{C p u}, \mathbf{M e m}$, Wid, Que).

(1) Node $=\left\{\right.$ Node $_{0}$, Node $_{1}$, Node $_{2}, \ldots$, Node $\left._{N-1}\right\}$ represents $N$ nodes in a cluster, $N \geq 1$.

(2) $\boldsymbol{C p u}=\left\{C_{0}, C_{1}, C_{2}, \ldots, C_{N-1}\right\}$ is a set of cluster node computing power, $C_{N-1}$ represents the computing power of Node $_{\mathrm{N}-1}$.

(3) $\boldsymbol{M e m}=\left\{M_{0}, M_{1}, M_{2}, \ldots, M_{N-1}\right\}$ is a set of cluster node memory resource, $M_{N-1}$ represents the memory size of Node $_{N-1}$.

(4) $\boldsymbol{W i d}=\left\{W_{0}, W_{1}, W_{2}, \ldots, W_{N-1}\right\}$ is a set of cluster node bandwidth size, $W_{N-1}$ represents the bandwidth size of Node $_{N-1}$.

(5) $Q u \boldsymbol{e}=\left\{Q_{0}, Q_{1}, Q_{2}, \ldots, Q_{N-1}\right\}$ is a set of cluster node waiting queue, $Q_{N-1}$ represents the waiting queue of Node $_{N-1}$.

Definition 2: The task set to be scheduled in the cluster can be represented as a graph $\mathbf{G}$. $\mathbf{G}=($ Task, Сотmu, Prop).

(1) $\boldsymbol{T a s k}=\left\{T_{0}, T_{1}, T_{2}, \ldots, T_{N-1}\right\}$ is a set of task node in a cluster, $N \geq 1 . T_{N-1}$ represents a task in the set, and can be divided into a set of sub tasks $\left\{t_{0}, t_{1}, t_{2}, \ldots, t_{N-1}\right\}$.

(2) $\mathbf{C o m m u}=\left\{\left(T_{i}, T_{j}\right)\right\}$ is a set of communication dependencies between task nodes in a cluster, in which $T_{i}, T_{j} \in \boldsymbol{T a s k}$, and the output of $T_{i}$ is the input of $T_{j}$. Similarly, communication dependencies of sub tasks can be defined as Commu'.

(3) $P r o p=\{I D$, Prio, VC, Mem, TS $\}$ is a quintet, represents the attribute set of the cluster task node.

$I D=\left\{I D_{0}, I D_{1}, I D_{2}, \ldots, I D_{N-1}\right\}$ is a set of serial number of task nodes in a cluster, $I D_{N-1}$ represents the serial number of $T_{N-1}$.

Prio $=\left\{P_{0}, P_{1}, P_{2}, \ldots, P_{N-1}\right\}$ is a set of task priority in a cluster, $P_{N-1}$ represents the priority of $T_{N-1}$. $\boldsymbol{V C}=\left\{V C_{0}, V C_{1}, V C_{2}, \ldots, V C_{N-1}\right\}$ is a set of computing resource demand for a task in a cluster, $V C_{N-1}$ represents computing resource demand of $T_{N-1}$.

$\boldsymbol{M e m}=\left\{M_{0}, M_{1}, M_{2}, \ldots, M_{N-1}\right\}$ is a set of memory resource demand for a task in a cluster, $M_{N-1}$ represents memory resource demand of $T_{N-1}$. 
$\boldsymbol{T S}=\left\{T S_{0}, T S_{1}, T S_{2}, \ldots, T S_{N-1}\right\}$ is the input requirement of resources for task execution, $T S_{N-1}$ represents input requirement of resources of $T_{N-1}$.

As shown in Figure 1, $T_{2}$ need resource of $T_{0}, T_{3}$ need resource of $T_{0}$ too, so the resource allocation of $T_{2}$ and $T_{3}$ will be after the allocation of $T_{0}$. But there's no communication dependency between $T_{0}$, $T_{1}, T_{5}$, they can be allocated in parallel. Similarly, $T_{2}, T_{3}$ can be allocated in parallel, $T_{4}$ will be after the allocation of $T_{2}$ and $T_{3}$.

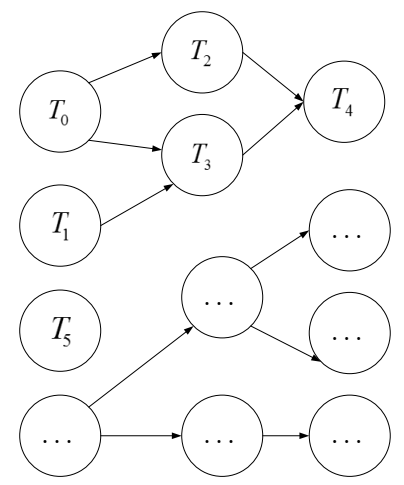

Figure 1.Schematic diagram of communication dependence relation

\subsection{Resource scheduling algorithm supporting network IO resource allocation}

In order to improve resource utilization rate and consider the efficient execution of tasks as far as possible under the environment of network congestion, this paper divides a set of $n$ tasks into $m$ sub task sets according to topological sort. After that, sub tasks will be allocated to appropriate cluster nodes according to their index from small to large, and tasks with data dependent will be allocated to the input data nodes or their nearby nodes, based on the greedy strategy and network awareness. As a result, the resource scheduling algorithm supporting network IO resource allocation can be expressed as follows:

$\left\{T_{0}, T_{1}, T_{2}, \ldots, T_{N-1}\right\} \rightarrow\left\{V_{0}, V_{1}, V_{2}, \ldots, V_{M-1}\right\}, V_{M-1}=\left\{T_{i}, T_{j}, \ldots, T_{k}\right\}$ is also a set of cluster tasks.

$V_{M-1}\left\{T_{i}, T_{j}, \ldots, T_{k}\right\} \rightarrow\left\{\right.$ Node $_{x}$, Node $_{y}, \ldots$, Node $\left._{z}\right\} \rightarrow<T_{i}$, Node $_{x}>$, which represents task $T_{i}$ is allocated to node Node $_{x}$.

\subsubsection{Partition of Task subset}

The basic idea of constructing task flow graph model based on topological sorting is to generate the topological sort of tasks by accurately describing the task size and quantity, and the data exchange between tasks. Based on the topological sequence and data interaction between tasks, it makes nondata interrelated tasks be decentralized and distributed to improve the utilization of the resources, while makes data interrelated taskshave greater probability to be assigned tosimilar nodes orregions having lower network load to speed up the completion.

Task scheduler will be triggered in the specific time and allocate tasks of the application to each node in the cluster, according to cluster resources and resources requirement of task running. In this way, all the tasks can be completed quickly and efficiently, and the utilization of cluster resources will be improved. The problem needs to meet the two constraints:

(1)When a node is allocated a task, the node should have sufficient computing resources and memory resources to run the task. The computing power of the cluster can be represented by the number of instructions per second.

(2)When a node is allocated a task, it should be ensured that the node have sufficient bandwidth for data exchanging with relate tasks' nodes, for accelerating the task completion and improve cluster throughput and resource utilization. 
Tasks of an application are modeled based on the topological sort of task flow graph. The modeldescribes all requests for an application as a directed acyclic graph (DAG)according to the tasks relationship of data exchanging. According to the topological sort algorithm, the task subset partition algorithm is shown in algorithm 1.

Algorithm 1: Task subset partition algorithm based on topological sort.

input: Directed acyclic graph $\mathbf{G}=($ Task, Commu, Prop)

output: Partitions of tasks: $V_{0}, V_{1}, V_{2}, \ldots, V_{M-1}$, for every $1 \leq i \neq j \leq M-1, V_{i} \cap V_{j}=\Phi$, and Task $=U_{i=1}^{M-1} V_{i}$

(1) Initialize $g$

(2) For $\left(\mathrm{i}=1 ; V_{i}=\Phi ; \mathrm{i}++\right)$ do

(3) Select $T_{j}$ from $G$ where In-degree $\left(T_{j}\right)=0, T_{j} \in$ Task

(4) Create $V_{i}=\left\{T_{j}\right\}$

(5) Delete $T_{j}$ from G, update Task $=$ Task $-V_{i}$

(6) End for

(7) Return $\left\{V_{0}, V_{1}, V_{2}, \ldots, V_{M-1}\right\}$

Thus, task set task can be divided to $\left\{V_{0}, V_{1}, V_{2}, \ldots, V_{M-1}\right\}$, and

(1) $T_{n} \in V_{m}, T_{n}$ can be allocated in parallel.

(2) $V_{n}, V_{m}$, if $n<m$, each task in $V_{n}$ should be allocated before tasks in $V_{m}$.

\subsubsection{Resource scheduling}

When the resource scheduler assigning tasks, it is according to topological sort results of the generated task flow graph. The basic idea of the network sensing resource allocation algorithm based on greedy strategy is that:

(1) When all topological sequences being initialized, task nodes with 0 degree will be discretely distributed to all nodes in the cluster, tasks with no data dependency will be giving CPU resources as much as possible, while data interrelated tasks will be allocated to the nodes having input data;

(2) When the following task nodes being allocated in the topological sequences, it need not only to ensure that data interrelated tasks are allocated to the same node or similar nodes, but also to ensure that tasks are allocated to nodes of low network load region.

3.2.2.1 Priority determination of cluster node

It is supposed that the number of effective nodes in the cluster is $N$, and the number of task nodes is $M$.

(1) To pre-configure calculation and memory resource matrix, in which the row vector represents the computing resources and memory resources, and the column vector represents $N$ cluster nodes;

(2) To get calculation and memory residual resource matrix, the row vector represents the remaining computation and memory resources, the column vector represents $N$ cluster nodes;

(3) To pre-configure the bandwidth resource matrix, the row vectors and the column vectors represent the $N$ nodes in the cluster, and the element BPre[s][d] represents the bandwidth from node $s$ to node $n$ for transmitting data;

(4) To get the network load matrix, the row and column vectors represent $N$ nodes in the cluster, the element Trans[s] [d] represents the file transfer size that has been allocated from node $s$ to node $d$;

(5) To get the resource matrix that has been allocated, among which the row vector represents the $M$ tasks, and the column vector represents the $N$ nodes in the cluster.

According to the network load matrix Trans $\left(N^{*} N\right)$ and the allocated resource matrix Allo $\left(M^{*} N\right)$, the cluster nodes are calculated to be assigned priority, and sorted according to the network priority. Priority sort algorithm pseudo code is shown as below.

Priority $[n]=$ null; //to store the priority

Cnt $0[n]=$ null; //to store the in-degree of task nodes

For $i$ from 1 to $\mathrm{n}$

For $j$ from 1 to $n$

$\operatorname{if}(i \neq j)$

Priority[i]=Priority[i] $>($ Trans[j] [i] /BPre[j][i] )? Priority[i] : (Trans[j][i] /BPre[j][i] );

End if 


\section{End for}

End for

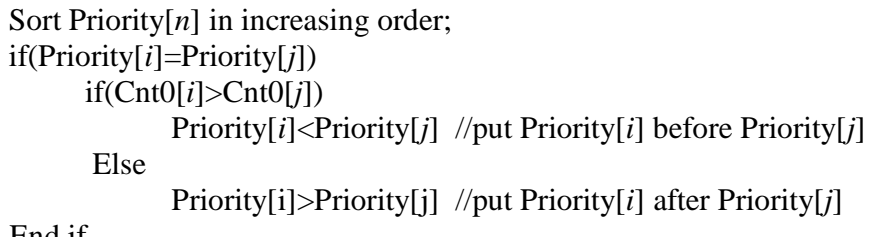

\subsubsection{Task allocation algorithm}

Topological sort algorithm based on the DSM matrix calculates the scheduling sequence, then chooses tasks and carries out tasks allocation in order, according to the priority sequence. In the process of scheduling, the recursive scheduling approach is adopted. Task scheduling is first carried out on task set $\boldsymbol{T a s k}=\left\{T_{0}, T_{1}, T_{2}, \ldots, T_{N-1}\right\}$, and then on sub task set $\left\{t_{0}, t_{1}, t_{2}, \ldots, t_{N-1}\right\}$. The Scheduling rule is that tasks should be allocated in the same or similar nodes, to reduce the cost of task communication. Task allocation algorithm flow can be shown in figure 2.

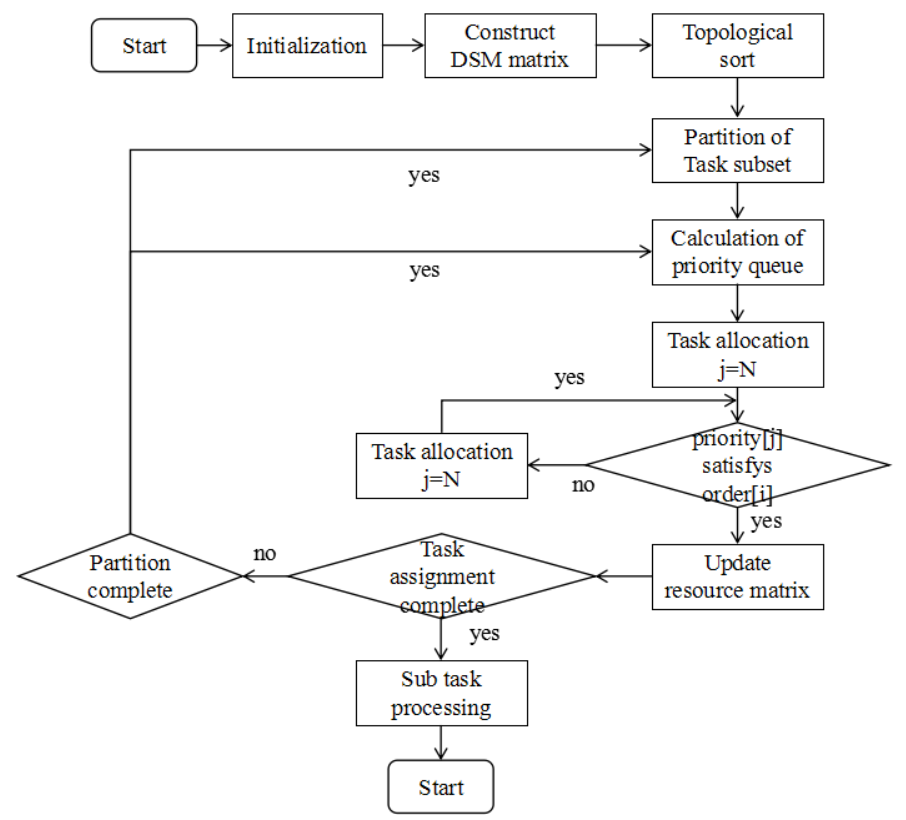

Figure 2. Task allocation algorithm flow

Task allocation algorithm flow is as following:

(1) Initial the task sequence Task;

(2) Construct DSM matrix;

(3) Calculate topological sort based on DSM;

(4) Calculate topological sort order [N] recursively;

(5) Calculate priority queue Priority[N] according to algorithm of 3.2.1;

(6) According to task set partition result $\left\{v_{1}, v_{2}, \ldots, v_{j}\right\}$, go through the topological sort order[N]. For each $\operatorname{order}[p]$, go through Priority[N], if satisfied, task of order [p] will be allocated on node with Priority $[q]$ and the resource matrix will be updated. Otherwise, set $q=q-1$;

(7) Return to (5), if there's task having not been allocated after processing task set $\boldsymbol{v}$.

(8) For sub task set $\left\{t_{0}, t_{1}, t_{2}, \ldots, t_{N-1}\right\}$, first step is to check whether the same node have enough resources. If it is, it will be assigned a task, otherwise, the nearest node will be chosen and checked until successfully assigned. 


\section{Performance evaluation}

200 hostsare supposed as nodes in the clusterin a virtualized resource pool for the simulation experiment of our algorithm. The host computing power is set as 100mips (millions of instructions per second), and the memory size is set as 8GB. In the simulation process, number of instructions for each task is set as 1024MI (millions of instructions). when idle nodes is randomly assigned to perform a number of tasks, there are two constraints in the implementation process need to be followed:

(1) On the same node, tasks closer to the top of topological sequence will be chosen and performed first;

(2) The start execution time of a task $i$ on node $d$ will not be earlier than the completion time of previous task on node $d$, and not earlier than the latest finish time plus data transmission time of any predecessor task of $i$ on any node except $d$.

As shown in figure 3, the improved YARN scheduling algorithm in this paper has lower execution time in the case of small network bandwidth, for taking the network resource into consideration. When the network bandwidth becomes larger, although the gap between the two algorithms becomes smaller, our scheduling algorithm is still better than the existing YARN resource scheduling algorithm.

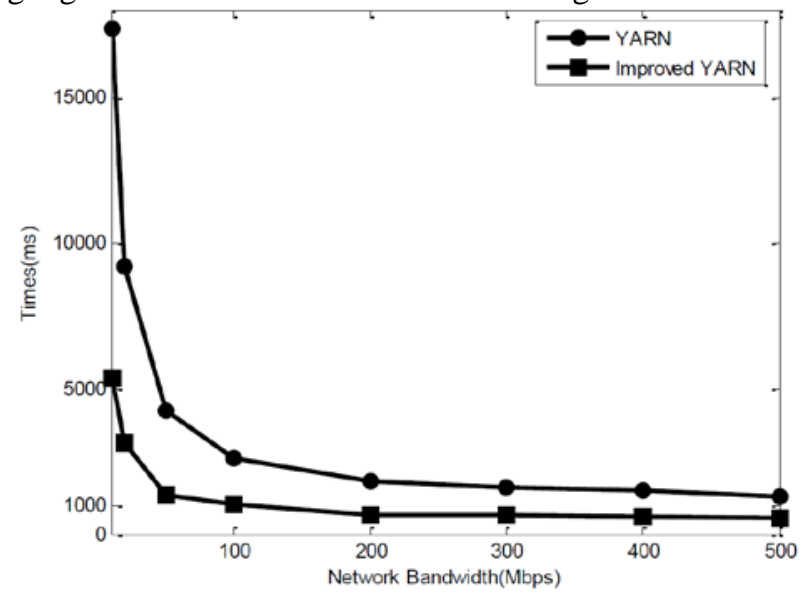

Figure 3. The task execution time of two algorithms

And as shown in figure 4, the improved YARN scheduling algorithm in this paper improves the CPU utilization obviously, owning to the decentralized distribution of non-data interrelated tasks and optimization of cluster resource.

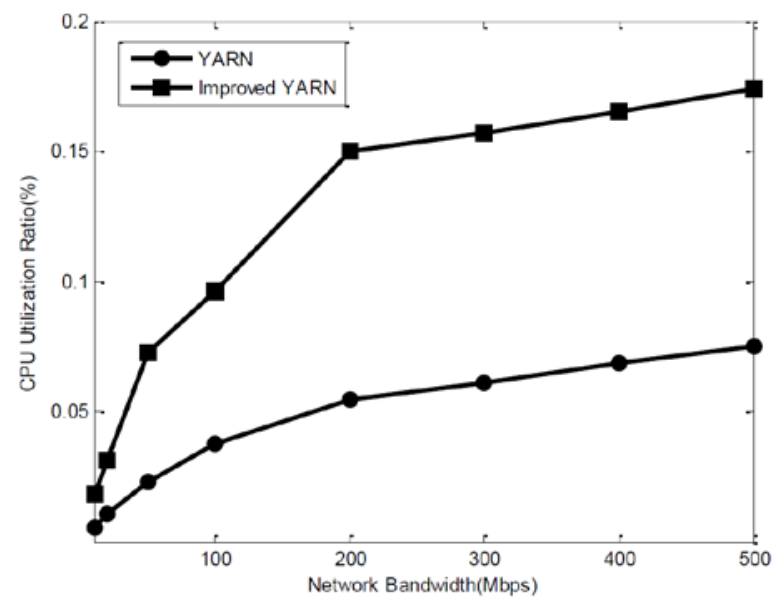

Figure 4. CPU utilization of two algorithms 


\section{Conclusion}

An improved YARN resource scheduling algorithm based on network load sensing is proposed in this paper. Through topological sorting task flow graph and network aware resource allocation algorithm, non-data interrelated tasks are decentralized and distributed so that the cluster resources are fully utilized, and data interrelated tasks are allocated based on same nodeor similar nodes with low network load which reduce theeffect, caused by network load, on the efficiency of task execution. We compare the algorithm presented in this paper with the existing YARN resources scheduling algorithm by simulation experiments, the results show that our algorithm has shortertask execution time and higher CPU utilization. Especially in the case of network congestion, the proposed algorithm can effectively improve the throughput and resource utilization.

\section{Acknowledgments}

This work was supported by the fundamental research funds for the central university, southwest university for nationalities (No.2015NZYQN28).

\section{References}

1. Solaimani M., Khan L., Thuraisingham B.: Real-time anomaly detection over VMware performance data using storm,Proceedings of the IEEE IRI 2014, 458-465(2014)

2. Fernandes, N. C. D., O.C.M.B.: XNetMon: A Network Monitor for Securing Virtual Networks,Proceedings of the ICC 2011,1-6(2011)

3. YaoY., JiayinW., Sheng Bo, JasonLin, NingfangMi: HaSTE: Hadoop YARN scheduling based on task-dependency and resource-demand,Proceedings of the 7th IEEE International Conference on Cloud Computing,184-191.0(2014)

4. Kapil B. S., Kamath S.S.: Resource aware scheduling in Hadoop for heterogeneous workloads based on load estimation. Proceedings of ICCCNT 2013, 1-5(2013)

5. Yao Y. W., Jiayin Sheng, Bo Lin, Jason Mi, Ningfang: Admission control in YARN clusters based on dynamic resource reservation,Proceedings of the 14th IFIP/IEEE International Symposium on Integrated Network Management, 838-841(2015)

6. Shieh A., Kandula S., Greenberg A., Kim C., Saha B.: Sharing the Data Center Network,NSDI, 309-322(2011)

7. Van Do, T. V., Binh T., Do Nam, Farkas Lóránt, Rotter Csaba, Tarjányi Támas: Building block components to control a data rate in the Apache Hadoop compute platform, Proceedings of the 18th International Conference on Intelligence in Next Generation Networks, 23-29(2015)

8. Yazdanov L., G. Maxim, Fetzer C.: EHadoop: Network I/O Aware Scheduler for Elastic MapReduce Cluster,Proceedings of the 8th IEEE International Conference on Cloud Computing, 821-828(2015)

9. Urgaonkar B., Shenoy P.: Sharc: Managing CPU and Network Bandwidth in Shared Clusters,IEEE Transaction on Parallel \& Distrributed Systems, 15(1): 2-17(2004)

10. Chen T.Y., WeiH. W., ChenM. F., et al.: LaSA: A locality-aware scheduling algorithm for Hadoop-MapReduce resource assignment,Proceedings of the 2013 International Conference on Collaboration Technologies and Systems, 342-346(2013)

11. Qin J., YonggangL., Mao X., McNair J.: Deadline based resource balancing task allocation for clustered heterogeneous LEO small satellite network. Proceedings of the 2013 IEEE Military Communications Conference, 1825-1831(2013)

12. Grandl R., A. Ganesh, Kandula S., Rao S., Akella A.: Multi-resource packing for cluster schedulers. Proceedings of the 2014 ACM Conference on Special Interest Group on Data Communication,455-466(2014) 